\title{
Editorial
}

\section{A Novel Treatment Strategy for Patients with Intermediate-Stage HCC Who Are Not Suitable for TACE: Upfront Systemic Therapy Followed by Curative Conversion}

\author{
Masatoshi Kudo
}

Department of Gastroenterology and Hepatology, Kindai University Faculty of Medicine, Osaka-Sayama, Japan

\section{Keywords}

Hepatocellular carcinoma $\cdot$ Intermediate-stage

hepatocellular carcinoma · Atezolizumab/bevacizumab

curative conversion

\section{Introduction}

Upfront systemic therapy with subsequent locoregional therapy is a new therapeutic option for intermediate-stage hepatocellular carcinoma (HCC). The first example of such treatment is sorafenib-transarterial chemoembolization (TACE) sequential therapy, which was tested in the TACTICS trial [1]. This was followed by lenvatinib-TACE (LEN-TACE) sequential therapy $[2,3]$, which is based on the high response rate $(40.6 \%$ per mRECIST) achievable with lenvatinib. A recently developed strategy is atezolizumab/bevacizumab curative (ABC) conversion, in which curative treatment such as resection, radiofrequency ablation (RFA), or superselective TACE (curative TACE) is performed after achieving marked tumor shrinkage (objective response rate [ORR] per RECIST v1.1 $=44 \%$ ) with atezolizumab plus bevacizumab (Atezo/Bev) combination therapy [4]. Given that approximately $20 \%$ of patients who undergo $\mathrm{ABC}$ con-

Masatoshi Kudo is Editor-in-Chief of Liver Cancer. karger@karger.com www.karger.com/lic

Karger $\stackrel{\text { ' }}{5}$

GOPEN ACCESS
(C) 2021 The Author(s).

Published by S. Karger AG, Basel

This is an Open Access article licensed under the Creative Commons Attribution-NonCommercial-4.0 International License (CC BY-NC) (http://www.karger.com/Services/OpenAccessLicense), applicable to the online version of the article only. Usage and distribution for commercial purposes requires written permission.

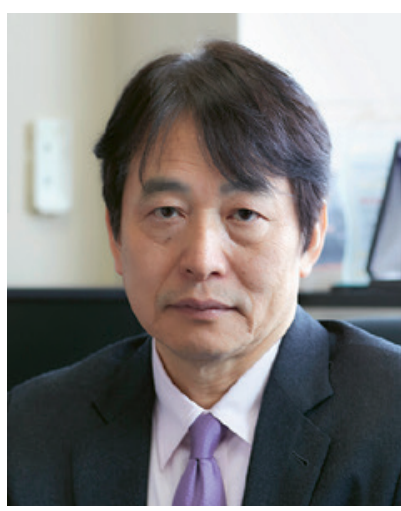

Prof. M. Kudo

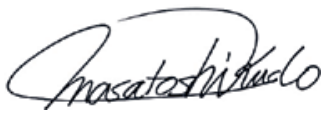

Editor Liver Cancer version therapy become cancer-free and drug-free, the optimal timing of curative conversion during Atezo/Bev treatment must not be missed, even when a sufficient effect of the drug is observed. Generally, the systemic treatment approach for other types of advanced cancer is "continue the treatment as long as the drug remains effective." However, curative conversion is frequently achievable using Atezo/Bev in patients with intermediate-stage HCC (locally advanced HCC without vascular invasion or extrahepatic spread), which is not possible in advanced HCC. The treatment concept that should be applied to intermediate-stage HCC is thus "switch to curative therapy to achieve cancer-free drug-free status upon

Correspondence to:

Masatoshi Kudo, m-kudo@med.kindai.ac.jp 
achievement of marked tumor shrinkage," which differs from the treatment concept for other types of advanced cancer or advanced HCC.

\section{Upfront Systemic Therapy with Subsequent Locoregional Therapy/Resection in Intermediate- Stage HCC}

TACE has been the standard of care for intermediatestage HCC until recently. However, this was based on evidence from a meta-analysis of 6 randomized controlled trials in which patients treated with TACE were compared with patients who did not receive therapy [5]. Although 6 effective drugs are currently available, the data are insufficient to determine whether TACE or upfront systemic therapy followed by selective TACE, ablation, or resection after tumor necrosis and/or shrinkage has a greater overall survival (OS) benefit.

Treatment with a vascular endothelial growth factor (VEGF) inhibitor decreases microvessel density, tumor interstitial pressure, and vascular permeability, in addition to normalizing the tumor vasculature, thereby improving drug delivery and the therapeutic efficacy of subsequent selective TACE [6]. Three types of sequential therapy in which locoregional therapy is preceded by systemic therapy have been reported: (1) sorafenib-TACE sequential therapy [1], (2) LEN-TACE sequential therapy $[2,3,7]$, and (3) ABC conversion therapy $[4,8]$.

This editorial comments on the 3 treatment strategies based on this completely new concept.

\section{Sorafenib-TACE Sequential Therapy}

The TACTICS trial (TACE Therapy In Combination with Sorafenib) was a multicenter randomized controlled study that examined progression-free survival (PFS) and OS in patients with HCC who underwent sequential therapy comprising upfront sorafenib with subsequent ondemand selective TACE and in those who underwent TACE alone [1]. The trial showed positive results: TACEspecific PFS, the primary endpoint, was 13.5 months in patients who underwent TACE alone versus 25.2 months in those who underwent sorafenib-TACE sequential therapy (hazard ratio $[\mathrm{HR}], 0.59 ; 95 \%$ confidence interval [CI], $0.41-0.87 ; p<0.0001$ ) [1]. Data of tumors within the up-to-seven criteria and those beyond the up-to-seven criteria were reported at the American Society of Clinical Oncology-Gastrointestinal Cancers Symposium in 2021
[9]. In patients with tumors beyond the up-to-seven criteria, PFS was 13.1 months longer with sorafenib-TACE sequential therapy than with TACE alone (22.1 months vs. 9.0 months; HR, 0.674). OS was also prolonged by 11.3 months by the addition of sorafenib to TACE ( 36.3 months vs. 25.0 months; HR, 0.898) [9]. In patients with tumors within the up-to-seven criteria, PFS was prolonged by 9.7 months with sorafenib-TACE sequential therapy compared with TACE alone (24.9 months vs. 15.2 months; HR, 0.756). OS was also prolonged by 3.7 months with sorafenib-TACE sequential therapy compared with TACE alone (35.6 months vs. 31.9 months; $\mathrm{HR}, 0.924)$. These data indicate that sorafenib-TACE sequential therapy is more effective than TACE alone in prolonging PFS and OS [9].

The results of the TACTICS trial support the use of sequential therapy comprising an upfront anti-VEGF inhibitor with subsequent selective TACE for intermediatestage HCC by demonstrating PFS and OS benefits.

\section{LEN-TACE Sequential Therapy}

LEN-TACE sequential therapy was developed in proof-of-concept studies based on the marked prolongation of OS in patients with intermediate-stage HCC beyond the up-to-seven criteria $[2,3]$. OS was 37.9 months with upfront lenvatinib with subsequent selective TACE versus 21.3 months with TACE alone, indicating that LEN-TACE sequential therapy significantly improved OS (HR, 0.48; 95\% CI, 0.16-0.79; $p<0.01$ ). PFS, ORR per mRECIST, and preservation of liver function were also favorable in the lenvatinib-treated group [3]. In addition, 5 of 30 patients (17\%) who received LEN-TACE sequential therapy achieved cancer-free drug-free status, indicating that this therapy can potentially achieve cure in patients with intermediate-stage HCC beyond the up-toseven criteria. The results were reproduced in many other clinical studies [7], and LEN-TACE sequential therapy has become a well-established approach for TACE-unsuitable intermediate-stage HCC in Japan. The idea that systemic therapy with a drug that achieves a high response (e.g., lenvatinib) combined with subsequent selective TACE for residual viable tumors increases the curative effect of TACE, preserves liver function, suppresses hypoxia-inducible cytokines, and ultimately improves survival in TACE-unsuitable intermediate-stage HCC led to the recommendation of this sequential therapy in the consensus statements of the Asia-Pacific Primary Liver Cancer Expert (APPLE) association [10] and the Japan 


\section{Atezolizumab+Bevacizumab Curative Conversion Therapy (ABC Conversion Therapy)}

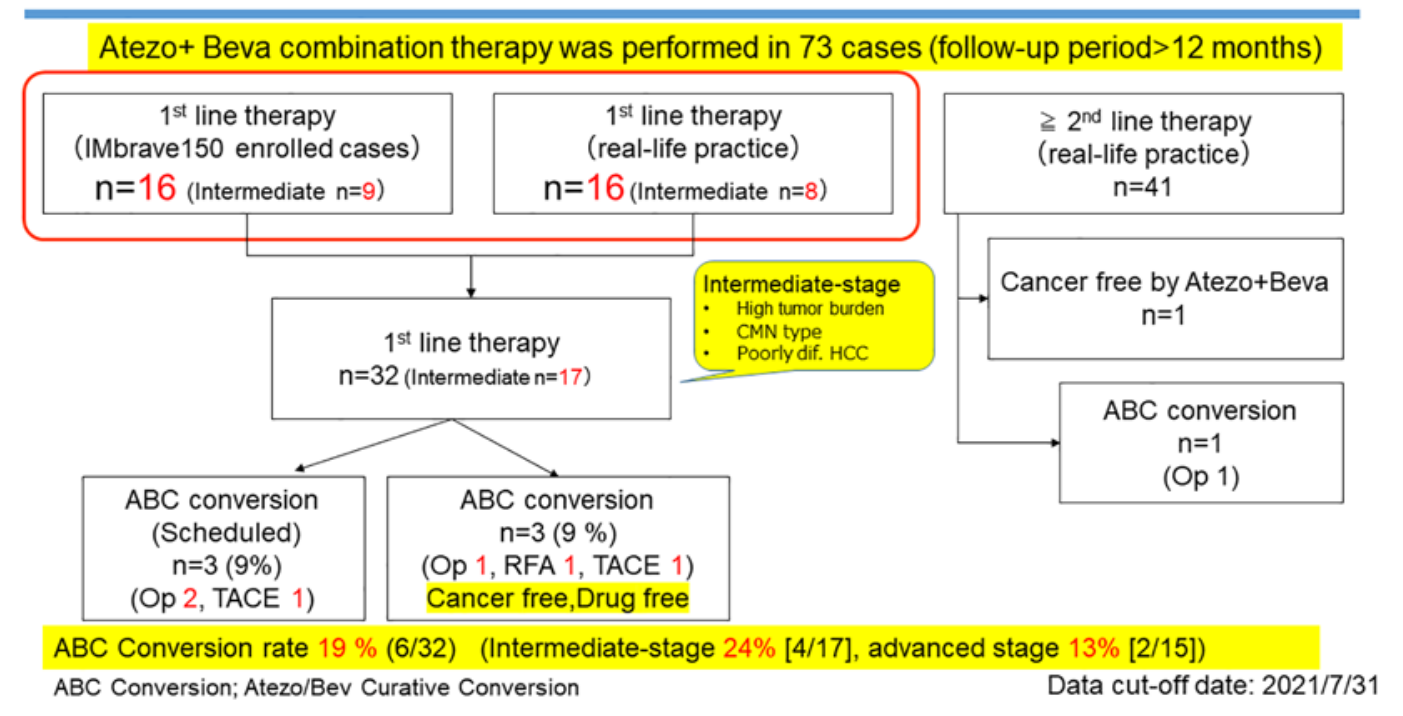

Fig. 1. ABC conversion therapy. Of 92 patients who received Atezo/Bev combination therapy, 73 were followed up for at least 12 weeks. Among them, 32 received atezolizumab plus bevacizumab as first-line treatment; 16 of them were enrolled in the IMbrave 150 trial. Of the 32 patients, 17 had intermediate-stage HCC, and ABC conversion (using resection, RFA, or TACE) was achieved in 3 of these 17 patients (9\%). When including the on-schedule cases (surgical resection in 2 cases and TACE in 1 case), curative conversion

Society of Hepatology (JSH) [11]. Thus, both the APPLE and JSH consensus statements recommend LEN-TACE sequential therapy as the first choice of treatment for TACE-unsuitable patients with intermediate-stage HCC $[10,11]$. Upfront systemic therapy was also recently recommended for TACE-unsuitable patients in the e-updated European Society for Medical Oncology clinical practice guidelines [12]. Further, the American Association for the Study of Liver Diseases consensus statement, which was updated in 2020, recommends upfront systemic therapy in addition to TACE in patients with intermediate-stage HCC with a high tumor burden [13]. This revision (i.e., systemic therapy as a treatment option for intermediate-stage HCC) is the first substantial revision in 20 years since the establishment of the Barcelona Clinic Liver Cancer algorithm in 1999.

Lenvatinib is associated with a high response rate per mRECIST, a quick response in TACE-resistant tumors such as confluent multinodular type or poorly differentiated HCCs, and a synergistic effect with TACE. Therefore, LEN-TACE sequential therapy has become an estab-

Upfront Systemic Therapy Followed by

Curative Conversion was achieved in approximately $19 \%$ of patients. When limited to 17 intermediate-stage patients, curative conversion was achievable in 4 patients (24\%), indicating that many patients can achieve cancer-free drug-free status and have a long survival with good quality of life (happy long survival). Cited from [4] with permission. HCC, hepatocellular carcinoma; TACE, transarterial chemoembolization; RFA, radiofrequency ablation; Atezo/Bev, atezolizumab plus bevacizumab.

lished treatment option that may lead to cancer-free drug-free status or longer survival even when complete response (CR) is not achieved in TACE-unsuitable intermediate-stage HCC.

\section{ABC Conversion Therapy}

Atezo/Bev combination therapy was approved worldwide in 2020 following the positive results of the Phase 3 IMbrave150 trial [14]. This therapy produces a high response rate in intermediate-stage HCC (i.e., ORR of $44 \%$ by RECIST 1.1) [15].

Figure 1 shows the results of 73 patients who were followed up for at least 12 weeks out of 92 patients and who received Atezo/Bev combination therapy (data cut-off date: July 31,2021 ) [4]. Of 32 patients who received the Atezo/ Bev therapy as first-line treatment, 3 (9\%) underwent curative conversion therapy ( $\mathrm{ABC}$ conversion therapy) (surgical resection, RFA, and curative TACE in 1 patient each), and the 3 patients achieved cancer-free drug-free status. Af- 

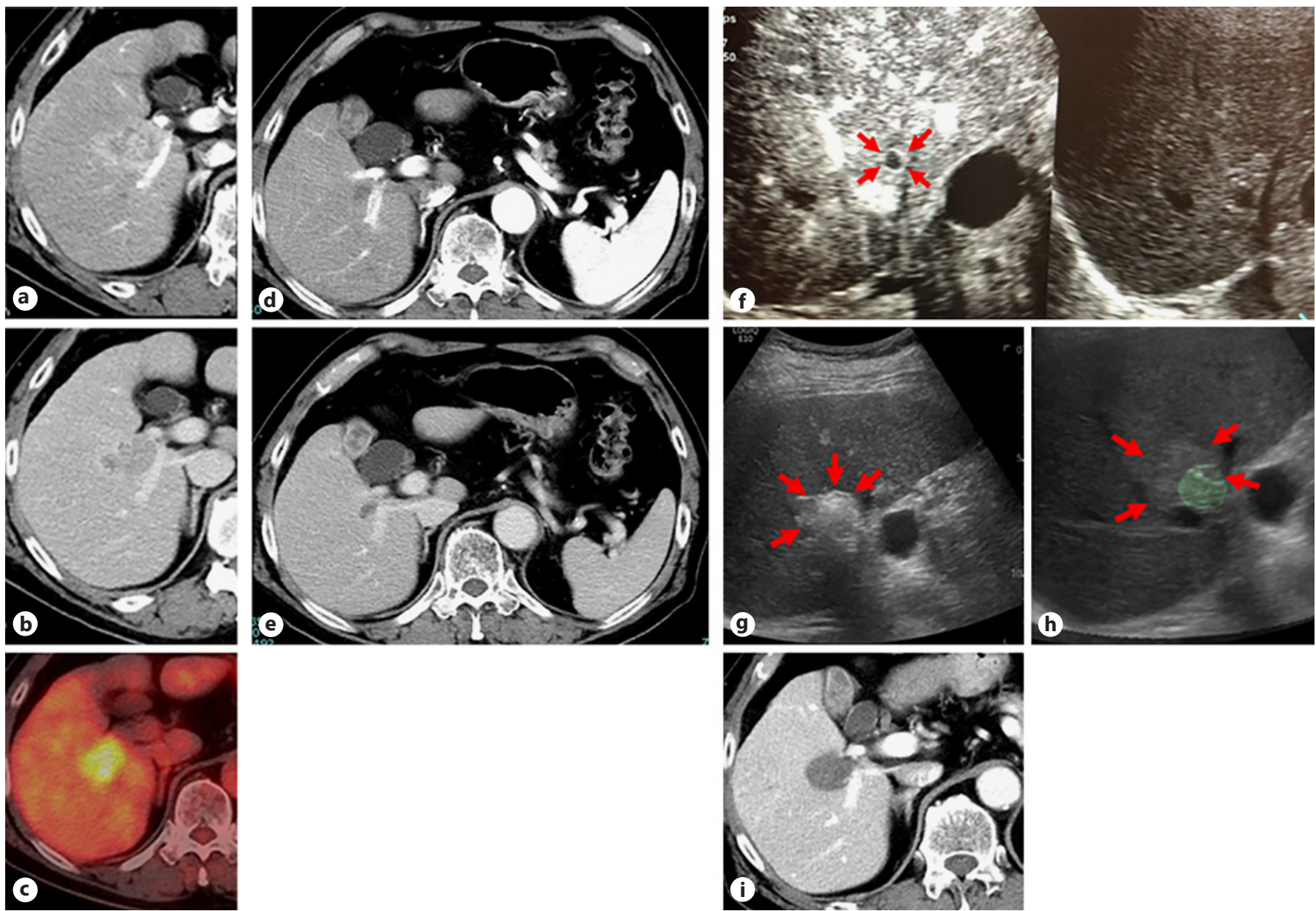

Fig. 2. An 80-year-old man who achieved cancer-free drug-free status by $\mathrm{ABC}$ conversion therapy. Confluent multinodular type HCC measuring $4.5 \mathrm{~cm}$ in diameter was confirmed at the porta hepatis area in the arterial phase (a) and the equilibrium phase of dynamic CT scan (b). It was considered incurable because ablation would pose a risk of bile duct injury. TACE was not considered effective in this case because of the tumor type, and the tumor was unresectable because the left lobe was extremely small. PET-CT showed intense FDG accumulation (c), indicating that the tumor was poorly differentiated HCC. After 8 cycles of Atezo/Bev combination therapy, the tumor showed a marked shrinkage of $67 \%$ in the arterial phase (d) and the equilibrium phase of dynamic CT

scan (e). Although viability was not evident on CT, contrast-enhanced ultrasound indicated residual blood flow in the ringshaped area (arrows) (f), indicating that only the peripheral area of the tumor remained viable. A bubble (arrows) formed after RFA $(\mathbf{g})$, in which the adequate ablative margin around the tumor was confirmed by US-US overlay fusion imaging (h). i CT performed 1 week after ablation confirmed complete response with enough ablative margin. To date, the patient remains cancer-free and drug-free. Cited from [4] with permission. HCC, hepatocellular carcinoma; RFA, radiofrequency ablation; Atezo/Bev, atezolizumab plus bevacizumab; ABC conversion, atezolizumab/bevacizumab curative conversion; TACE, transarterial chemoembolization.

ter including 2 cases of scheduled surgical resection and 1 case of scheduled curative TACE, the curative conversion rate becomes relatively high (19\%). Moreover, if the analysis is limited to 17 intermediate-stage cases, the curative conversion rate is much higher (24\%, Fig. 1). Unlike molecular targeted agents, Atezo/Bev combination therapy produces marked tumor shrinkage in responders, even in those with highly malignant PET-positive HCC such as confluent multinodular type or poorly differentiated HCC.

Consequently, surgical resection, ablation, or curative TACE becomes feasible, and pathological CR and drug-free status can be achieved in $20-30 \%$ of cases (Fig. 2, 3).

In the oncology field, once a systemic therapy is initiated, it will be continued if the systemic agent achieves a response of stable disease or better. This is particularly true when a partial response (PR) is achieved; in these cases, the therapy is not changed as long as PR continues. However, in HCC, especially in intermediate-stage HCC 


\section{Treatment Strategy for Intermediate-stage HCC 2021}

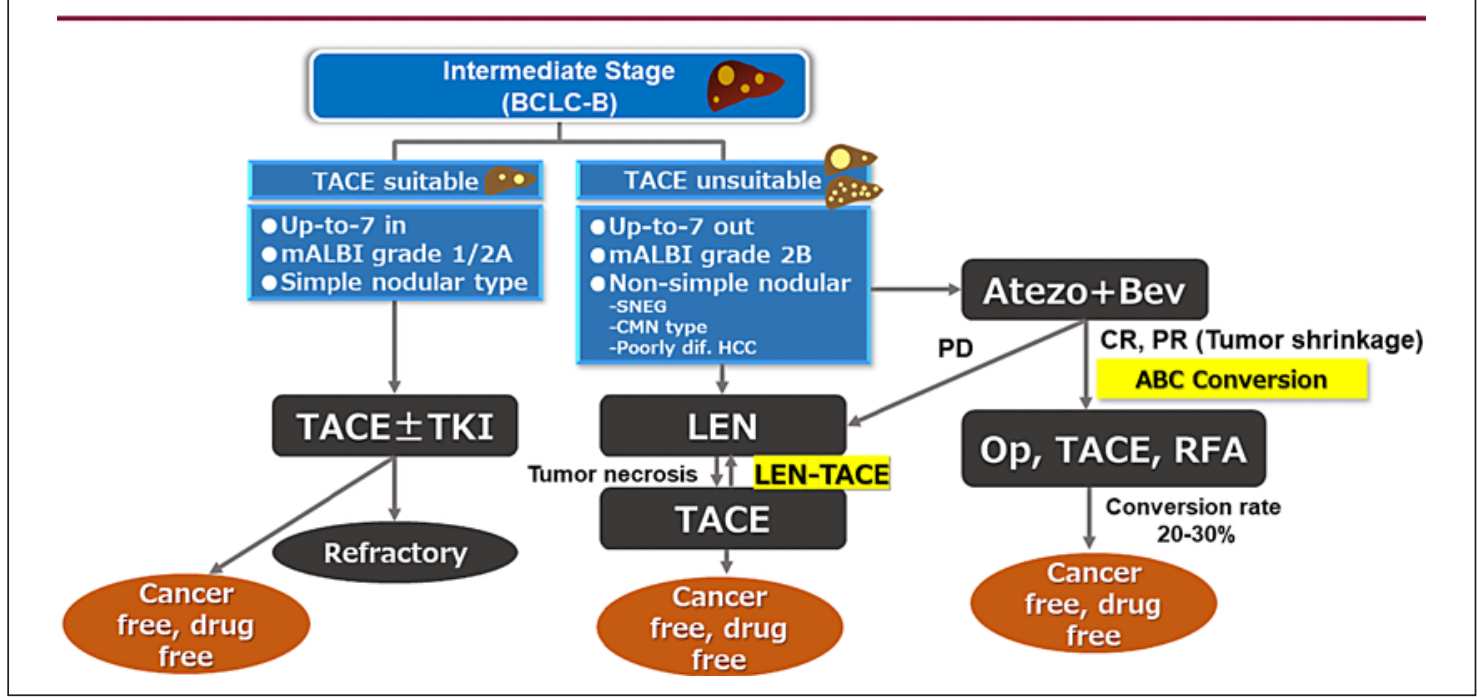

Fig. 3. Novel treatment strategy for intermediate-stage HCC 2021. Cited from [4] with permission. Curative conversion rate by $\mathrm{ABC}$ conversion was $20-30 \%$. mALBI grade, modified albumin-bilirubin grade; SNEG, simple nodular type with extra growth; CMN, confluent multinodular type; LEN, lenvatinib; ABC conversion, atezolizumab/bevacizumab curative conversion; HCC, hepatocellular carcinoma; RFA, radiofrequency ablation; PD, progressive disease; $\mathrm{CR}$, complete response; PR, partial response; TACE, transarterial chemoembolization.

cases that remain locally advanced without vascular invasion or extrahepatic spread, other curative treatments are available, such as ablation or curative TACE in addition to resection, once marked tumor shrinkage is achieved. Thus, continuing the systemic therapy is not a good strategy. When a deep tumor response (marked tumor shrinkage) is achieved using Atezo/Bev combination therapy, curative conversion, rather than continuing sequential systemic therapy, must therefore be implemented without missing the best possible timing (i.e., while the deep response is maintained). The marked survival benefit in patients who achieved curative treatment is well known. Thus, for intermediate-stage HCC, the use of systemic therapy is based on a concept that is completely different from that of conventional sequential therapies in cases of advanced-stage HCC. In other words, systemic therapy should be introduced to achieve tumor shrinkage as induction therapy for curative conversion therapy.

An ORR of 44\% per RECIST v1.1 was achieved using Atezo/Bev combination therapy in patients with intermediate-stage HCC [15]. This means that nearly one in two of these patients can potentially achieve curative conversion. Thus, in patients with intermediate-stage HCC receiving Atezo/Bev combination therapy, curative conversion must be implemented as soon as possible without hesitation upon achieving a sufficiently deep response instead of continuing the systemic therapy until progressive disease; real cure is not expected at this point. A pathological CR is rarely achieved with systemic treatment alone (e.g., lenvatinib or Atezo/Bev); residual viable cancer is often found when resection is performed in patients who seemed to achieve CR according to mRECIST. In such cases, recurrence is most likely to occur when the systemic therapy is discontinued; to prevent this, curative conversion must be implemented whenever possible, even if imaging findings indicate CR or deep response. Of note, in patients who undergo resection as conversion surgery, bevacizumab needs to be discontinued at least 4-6 weeks before the procedure to prevent bleeding event, whereas a 3-week interval is sufficient when performing ablation or curative TACE. In either case, ABC conversion can be achieved in approximately $20-30 \%$ of patients with intermediate-stage HCC, as shown in Figure 1.

\section{Conclusion}

This editorial describes a new treatment concept for intermediate-stage HCC. The TACTICS trial demonstrated that a sequential approach consisting of upfront 
systemic therapy using a drug with anti-VEGF activity followed by selective TACE improves PFS and OS in cases beyond the up-to-seven criteria and provides a benefit to some extent in cases within these criteria. This was confirmed by the improvement of OS achieved with LENTACE sequential therapy comprising first-line lenvatinib with subsequent selective TACE. LEN-TACE sequential therapy is now established and often used in daily clinical practice in Japan.

Atezo/Bev combination therapy induces tumor shrinkage unlike other targeted therapies, and this tumor shrinkage can be achieved even in patients with intermediate-stage HCCs with a high malignant grade, such as poorly differentiated HCC. If curative conversion (i.e., conversion to resection, RFA, or curative TACE) is implemented with optimal timing during best response, approximately $20-30 \%$ of patients can achieve cancer-free and drug-free status. Patients who remain drug-free for a long time do not have adverse events and are expected to have a long survival with good quality of life ("happy long survival"). If there is a recurrence, it can be detected at an early stage when many treatment options are still available and liver function is maintained, and thus curative therapy can be applied again.
This is another reason why we should recognize that the concept of systemic therapy for intermediate-stage HCC is different from that for advanced HCC or other types of solid cancer. The treatment goal in intermediatestage HCC should be to "achieve cure (cancer-free drugfree status) whenever possible."

\section{Conflict of Interest Statement}

The author received lecture fees from Eisai, Bayer, MSD, BMS, EA Pharma, Eli Lilly, and Chugai; grants from Eisai, Takeda, Otsuka, Taiho, EA Pharma, Gilead Sciences, AbbVie, Sumitomo Dainippon Pharma, Chugai, and Ono Pharma; advisory consulting fees from Eisai, Ono, MSD, BMS, and Roche. Masatoshi Kudo is the Editor-in-Chief of Liver Cancer.

\section{Funding Sources}

None.

\section{Author Contributions}

M. Kudo conceived, wrote, and approved the final manuscript.

\section{References}

1 Kudo M, Ueshima K, Ikeda M, Torimura T, Tanabe N, Aikata H, et al. Randomised, multicentre prospective trial of transarterial chemoembolisation (TACE) plus sorafenib as compared with TACE alone in patients with hepatocellular carcinoma: TACTICS trial. Gut. 2020 Aug;69(8):1492-501.

2 Kudo M. A new treatment option for intermediate-stage hepatocellular carcinoma with high tumor burden: initial lenvatinib therapy with subsequent selective TACE. Liver Cancer. 2019;8:299-311.

3 Kudo M, Ueshima K, Chan S, Minami T, Chishina $\mathrm{H}$, Aoki $\mathrm{T}$, et al. Lenvatinib as an initial treatment in patients with intermediate-stage hepatocellular carcinoma beyond up-to-seven criteria and Child-Pugh A liver function: a Proof-of-Concept Study. Cancers. 2019 Jul 31;11(8):1084.

4 Kudo M, Aoki T, Ueshima K, Nishida N. Upfront systemic therapy with subsequent curative conversion for intermediate-stage hepatocellular carcinoma: atezolizumab + bevacizumab curative conversion (ABCConversion) therapy. KanTanSui. 2021;83:475-783.

5 Llovet JM, Burroughs A, Bruix J. Hepatocellular carcinoma. Lancet. 2003 Dec 6; 362(9399):1907-17.
6 Jain RK. Normalization of tumor vasculature: an emerging concept in antiangiogenic therapy. Science. 2005 Jan 7;307(5706):58-62.

7 Kawamura Y, Kobayashi M, Shindoh J, Kobayashi Y, Okubo S, Tominaga L, et al. Lenvatinib-transarterial chemoembolization sequential therapy as an effective treatment at progression during lenvatinib therapy for advanced hepatocellular carcinoma. Liver Cancer. 2020;9(6):756-70.

8 Kudo M. A new era in systemic therapy for hepatocellular carcinoma: atezolizumab plus bevacizumab combination therapy. Liver Cancer. 2020 Apr;9(2):119-37.

9 Kudo M, Ueshima K, Ikeda M, Torimura T, Tanabe M, Aikata H, et al. TACTICS: final overall survival data from a randomized, open label, multicenter, Phase II trial of transcatheter arterial chemoembolization (TACE) therapy in combination with sorafenib as compared with TACE alone in patients with hepatocellular carcinoma. 2021. ASCO-GI.

10 Kudo M, Han KH, Ye SL, Zhou J, Huang YH, Lin SM, et al. A changing paradigm for the treatment of intermediate-stage hepatocellular carcinoma: Asia-Pacific primary liver cancer expert consensus statements. Liver Cancer. 2020 Jun;9(3):245-60.
11 Kudo M, Kawamura Y, Hasegawa K, Tateishi R, Kariyama K, Shiina S, et al. Management of hepatocellular carcinoma in Japan: JSH consensus statements and recommendations 2021 update. Liver Cancer. 2021 Jun;10(3): 181-223.

12 Vogel A, Martinelli E, Vogel A, Cervantes A, Chau I, Daniele B, et al. Updated treatment recommendations for hepatocellular carcinoma (HCC) from the ESMO clinical practice guidelines. Ann Oncol. 2021 Jun;32(6):801-5.

13 Llovet JM, Villanueva A, Marrero JA, Schwartz M, Meyer T, Galle PR, et al. Trial design and endpoints in hepatocellular carcinoma: AASLD consensus conference. Hepatology. 2020 May 20:158-91.

14 Finn RS, Qin S, Ikeda M, Galle PR, Ducreux M, Kim TY, et al. Atezolizumab plus bevacizumab in unresectable hepatocellular carcinoma. N Engl J Med. 2020 May 14;382(20): 1894-905.

15 Finn R, Qin S, Ikeda M, Galle P, ducreux M, Kim TY, et al. IMbrave150: updated overall survival (OS) data from a global, randomized, open-label Phase III study of atezolizumab (atezo) + bevacizumab (bev) vs sorafenib (sor) in patients (pts) with unresectable hepatocellular carcinoma (HCC). EASL Digital Liver Cancer Summit. 2021 Feb 5-6:2021. 